\title{
On V603 Aql and Magnetic Novae
}

\section{A. Schwarzenberg-Czerny ${ }^{1,2}$ and A. Udalski ${ }^{1,3}$}

1 Warsaw University Observatory, Al. Ujazdowskie 4, 00- 478 Warsaw, Poland.

2 Astronomisches Institut der Universität Münster, Wilheln-Klemm-Str. 10, 4400 Münster, F.R.G. 3 York University, 4700 Keele Street, North York, Ontario, Canada M3J P3.

Our photometry revealed that the old nova V603 Aql has four different coherent periods, including a period present in X-rays and so it is quite unique among all novae. Thus we classify V603 Aql as an intermediate polar (IP) system. Some time ago Hefner and Metz (1985) found that the period of the sinusoidal light variations of $\mathrm{V} 603 \mathrm{Aql}$ is 3.5 hour and thus it is longer by about 5 percent than the spectroscopic orbital period of 3.3 hour. Our observations covering over 30 bours and spanning 30 days confirm their result. However, we discovered also another coherent oscilation with a small amplitude of $0.02 \mathrm{mag}$ and period of $61 \mathrm{~min}$ (Udalski and Schwarzenberg-Czenas. 1989). Our analysis of the X-ray observations by Drechsel et al. (1983) revealed 25 percent motulation with the same period and thus confirmed our discovery (Fig. 1). Additionaly we found in our light curves evidence of the 2.5 day beat period of the 3.3 and 3.5 hour periods.

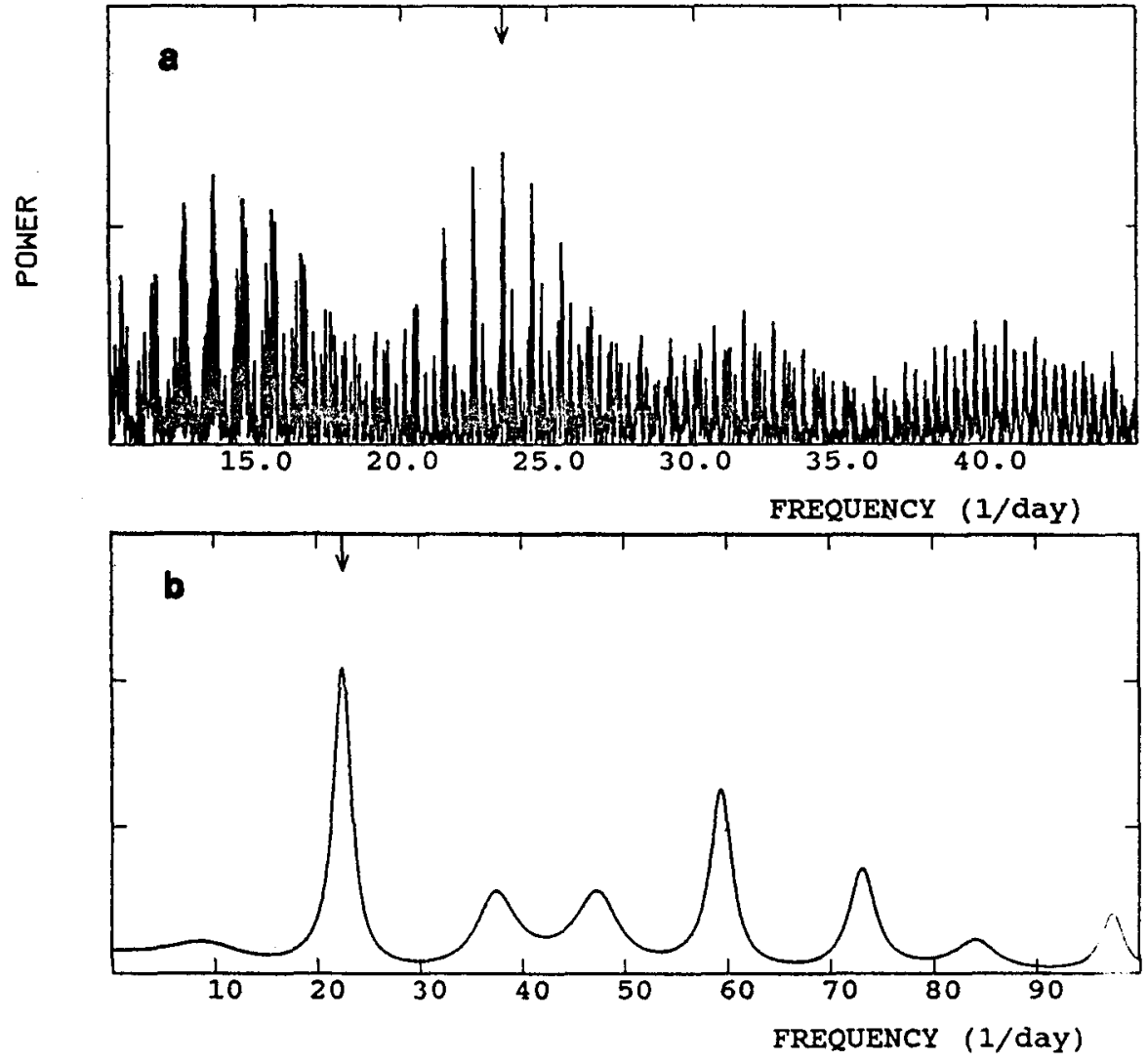

Fig. 1. (a) Power spectrum of V603 Aql light curve after prewhitening of the 3.5 hour period. Main features correspond to the 1.75 hour harmonic and to the 61.4 min period, marked with an arrow on both plots. (b) Maximum entropy power densily function for Drechsel et al. (j983) X-ray observations. 
Table 1. Intermediate polars and novae with known orbital periods.

\begin{tabular}{|l|r|rr|r|c|}
\hline $\begin{array}{l}\text { Object } \\
\text { narne }\end{array}$ & $\begin{array}{r}\text { Orbital } \\
\text { period [d] }\end{array}$ & $\begin{array}{c}\text { Interm. } \\
\text { Polar }\end{array}$ & Nova & $\begin{array}{r}\text { Rate of } \\
\text { decline } t_{3}\end{array}$ & Reference \\
\hline GK Per & 1.996 & $\mathrm{v}$ & $\mathrm{v}$ & 13 & 1,2 \\
V795 Her & .615 & $\mathrm{v}$ & & & 1 \\
DI Lac & .543 & & $\mathrm{v}$ & 43 & 1,2 \\
V1668 Cyg & .439 & & $\mathrm{v}$ & 23 & 1,2 \\
AE Aqr & .411 & $\mathrm{v}$ & & & 1 \\
BT Mon & .334 & & $\mathrm{v}$ & fast & 1,2 \\
V533 Her & $.28:$ & $\mathrm{v}$ & $\mathrm{v}$ & 44 & 1,2 \\
H0542-407 & .258 & $\mathrm{v}$ & & & 1 \\
V426 Oph & .250 & $\mathrm{v}$ & & & 1 \\
TV Col & .228 & $\mathrm{v}$ & & & 1 \\
HR Del & .214 & & $\mathrm{v}$ & 230 & 1,2 \\
T Aur & .204 & & $\mathrm{v}$ & 100 & 1,2 \\
DQ Her & .194 & $\mathrm{v}$ & $\mathrm{v}$ & 94 & 1,2 \\
FO Aqr & .167 & $\mathrm{v}$ & & & 1 \\
WY Sge & .154 & & $\mathrm{v}$ & $?$ & 1,2 \\
AO Psc & .149 & $\mathrm{v}$ & & & 1 \\
RR Pic & .145 & $\mathrm{v}$ & $\mathrm{v}$ & 150 & 1,2 \\
V1223 Sgr & .140 & $\mathrm{v}$ & & & 1 \\
V1500 Cyg & .139 & $\mathrm{v}$ & $\mathrm{v}$ & 3.6 & 1,2 \\
V603 Aql & .138 & $\mathrm{v}$ & $\mathrm{v}$ & 8 & $1,2,3$ \\
BG CMi & .134 & $\mathrm{v}$ & & & 1 \\
V Per & .107 & & $\mathrm{v}$ & & 2,4 \\
EX Hya & .068 & $\mathrm{v}$ & & & 1 \\
CP Pup & .061 & & $\mathrm{v}$ & 8 & $1, \dot{2}$ \\
GQ Mus & .059 & $\mathrm{v}:$ & $\mathrm{v}$ & 45 & 2,5 \\
SW UMa & .056 & $\mathrm{v}$ & & & 1 \\
\hline
\end{tabular}

References: (1) Ritter, 1987; (2) Duerbeck, 1989; (3) this paper; (4) Shafter, 1939; (5) Diaz and Steiner, 1989.

Again, we found an independent confirmation of our discovery in the data obtained by Haefner and Metz (1985).

Among CVs only TV Col resembles V603 Aql, having periods of $32 \mathrm{~min}, 5.5$ and 5.3 hour and 4 days. We suggest that the same physical mechanism works in both systems. Their neutron binary counterparts are $\mathrm{HZ} \mathrm{Her}$ and SS433. By analogy to TV Col we identify the 61 min period with the rotation period of the white dwarf. Free precession of the white dwarf is no likely explanation of the 2.5 day period because of its shortness and white dwarf's large momentum of inertia. More likely are the mechanisms involving the accretion disc. One such mechanism, namely precession of the tilted accretion disc was proposed by Bonnet-Bidaud et al. (1985) for TV Col. If the similarity between TV Col and V603 Aql on the one side and HZ Her and SS433 on the other side is not superficial than free precession of the magnetic neutron star is no likely explanation of the latter systems.

Thus V603 $\Lambda \mathrm{q} l$ turned another IP magnetic system among novae. The question arises whether a casual relation exists among novae and magnetic CV systems. We shall investigate this point further from the statistical point of view. All intermediate polars and novae with known orbital periods are listed in Table 1. Inspection of the table reveals that at least half of the novae are magnetic systems. In fact, their relation goes deeper. In Fig. 2 we plot the cumulative period distributions for intermediate polars (IPs) and novae (N), based on Table 1. The two distributions resemble each other. Indeed, two statistic tests revealed no significant differences among these empirical 


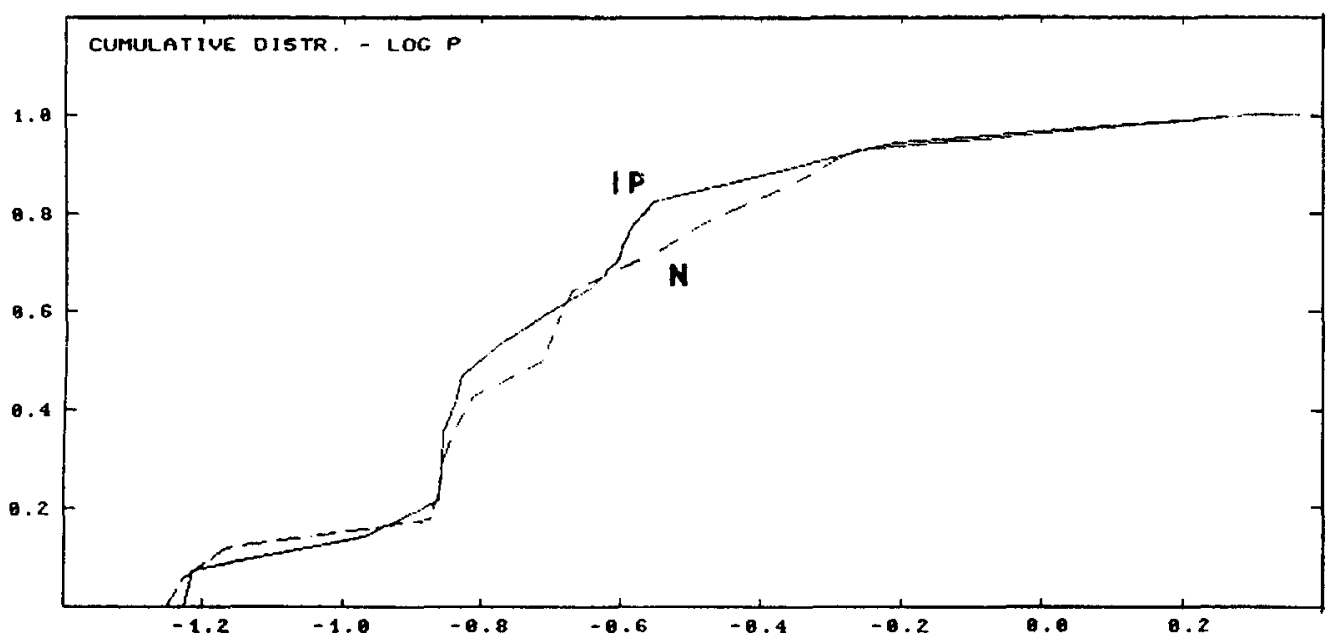

$109 P$

Fig. 2. The cumulative distributions of the log orbital periods (days) of novae (N) and intermediate polars (IP). The two distributions do not differ statisticaly (see text).

distributions at confidence level 0.95 . The tests applied were the mean of a sample $(\mathrm{N})$ from a finite population $(N \cup I P)$ test (liendall and Stuart, 1976) and the analysis of variance test for three classes $(N-I P, N \cap I P, I P-N)$. As usualy, this result must be treated with care, since lack of evidence is no evidence of lack. We found no obvious correlation among the speed class and magnetic properties.

We conclude by stressing three points. Firstly current models of outbursts which ignore magnetic fields apply to less thien balf of novae only. Secondly, full three dimensional computations are required in order to model the magnetic novae in which the rotation and magnetic axes are tilted. Finaly, we urge further investigations in order to clarify whether all novae are magnetic.

\section{References}

Bonnet-Bidaud, J.M., Motch, C., Mouchet, M., 1985, Astron. Astrophys., 143, 313.

Diaz, M.P. and Steiner, J.E., 1989, Astroph. J., 339, L41.

Drechsel, H., Rahe, J., Seward, F.D., Wang, Z.R. and Wargau, W., 1983, Astron. Astrophys., $126,357$.

Duerbeck, H., 1987, em A reference Catalogue and Atlas of Galactic Novae, Reidel, Dordrecht.

Haefner, R. and Metz, K., 1985, Astron. Astrophys., 145, 311.

Kendall, M. and Stuart, A., 1976, The Advanced Theory of Statistics, 3, 171, Griffin, London.

Ritter, H., 1987, Astron. Astrophys. Supp., 70, 335.

Shafter, A., 1989, Astroph. J., 339, L75.

Udalski, A. and Schwarzenberg-Czerny, A., 1989, Acta Astron., 39, 125. 